REVISIÓN DE LITERATURA

\title{
RESPUESTA PRODUCTIVA DEL GANADO LECHERO ANTE EL SUMINISTRO DE METIONINA SINTÉTICA ${ }^{1}$
}

Omar Andrés Vargas-Villalobos ${ }^{2}$, Jorge Alberto Elizondo-Salazar ${ }^{2}$

\section{RESUMEN}

La nutrición animal ha venido siendo cada vez más precisa con el objetivo de lograr la máxima eficiencia al menor costo y el menor impacto ambiental. Los aminoácidos son requeridos sobre un patrón ideal para cubrir las necesidades fisiológicas de los animales en las diferentes etapas de vida. Los rumiantes pueden aprovechar aminoácidos esenciales de la microflora ruminal, sin embargo ésta es insuficiente para llenar los requerimientos de vacas altas productoras. La utilización de aminoácidos sintéticos para llenar las deficiencias de metionina y lisina en la alimentación de vacas lecheras, que consumen alimentos con altas proporciones de granos, se presenta como una opción viable, ya que se ha comprobado la baja presencia de estos dos aminoácidos en donde la fuente de proteína proviene de derivados del maíz y la harina de soya. También se ha demostrado que la cantidad de metionina y lisina excretados por la glándula mamaria es mayor que la de otros aminoácidos, además todo parece indicar que la metionina posee una alta tasa de degradación en el rumen. La utilización de altas cantidades de proteína cruda en las raciones puede provocar una alta excreción de nitrógeno ureico al ambiente aparte de que puede ser perjudicial para la salud del animal, sin garantizar que se estén llenando los requerimientos de aminoácidos. Algunos de los resultados obtenidos con la suplementación de aminoácidos sintéticos son incremento en producción, incremento en rendimiento de grasa y proteína láctea y disminución en la excreción de nitrógeno al ambiente. El objetivo de esta revisión literaria es dar a conocer el efecto de la suplementación de metionina en la alimentación de vacas lecheras altas productoras.

Palabras clave: aminoácidos, sólidos lácteos, nitrógeno, aditivo nutricional.

\footnotetext{
${ }^{1}$ Inscrito en Vicerrectoría de Investigación. Universidad de Costa Rica. Proyecto 737-B5-188. ${ }^{2}$ Universidad de Costa Rica. Estación Experimental Alfredo Volio Mata. Facultad de Ciencias Agroalimentarias.

Autores para correspondencia: omandres1219@gmail.com, jorge.elizondosalazar@ucr.ac.cr Recibido: 12 enero 2015 
Dairy cattle production response to the provision of synthetic methionine. Animal nutrition has become more precise in order to achieve maximum efficiency at lower cost and less environmental impact. Amino acids are required in an ideal pattern to meet the physiological needs of animals at different stages of life. Ruminants can take advantage of essential amino acids of rumen microflora; however it is insufficient to meet the requirements of high producing cows. The use of synthetic amino acids to fill the deficiencies of methionine and lysine in the diet of dairy cows consuming diets with a high proportion of grains is presented as a viable option, since it has been proven low presence of these two amino acids, especially when protein source comes from corn and soybean meal. It has also been shown that the amount of methionine and lysine excreted by the mammary gland is greater than that of other amino acids; in addition, it also appears that methionine has a high rate of degradation in the rumen. The use of high amounts of crude protein in the rations can cause high urea nitrogen excretion into the environment and it can be detrimental to the health of the animal, with no warranty that the amino acid requirements are filled. Some of the results obtained with synthetic amino acid supplementation are increased production, increased yield of fat and milk protein and decreased excretion of nitrogen to the environment. The purpose of this literature review is to show the effect of supplementation of methionine in the diet of high producing dairy cows.

Key words: amino acids, milk solids, nitrogen, nutritional additive

\section{INTRODUCCIÓN}

Incrementar el contenido de sólidos lácteos es un objetivo importante para los productores de leche y para lograrlo se requiere de un adecuado suministro postruminal de aminoácidos (AA).

En el caso del ganado lechero, la metionina (Met) y la lisina (Lis) se consideran los aminoácidos esenciales para la síntesis de la leche y la proteína en ella (Patterson y Kung 1988, Piepenbrink et al. 1996, Xu et al. 1998, Chen et al. 2011), debido a que la concentración de Lis y Met excretada por la glándula mamaria, es mayor que la de los otros aminoácidos (Bremmer et al. 1997). 
La Met también se considera limitante cuando la dieta se compone de pequeñas cantidades de maíz, altas cantidades de forraje o cuando las fuentes de proteína de sobrepaso se componen de productos derivados de la soya (Rulquin et al. 2006).

Chen et al. (2011) realizaron un estudio con el objetivo de cuantificar la respuesta de la producción láctea supliendo diferentes fuentes de metionina absorbible. En dicho estudio utilizaron isopropil-2-hidroxi-4-(Metiltio)-ácido butanoico ( $\mathrm{HMBi}$ ) y metionina protegida al rumen (MPR) ajustando la concentración de Lis y Met para mantener una relación 3:1. Los autores determinaron que alimentar con $\mathrm{HMBi}$ incrementó el rendimiento lácteo, el contenido proteico y los sólidos no grasos. En la misma investigación se comprobó que al suplementar con 0,06\% de MPR y una concentración de $15,5 \%$ de proteína cruda (PC), la eficiencia de utilización de nitrógeno fue mayor que cuando se alimentó con una dieta que contenía 16,8\% de PC sin fuentes de metionina.

Considerando que la PC es uno de los nutrientes más caros en las dietas para ganado de leche, el objetivo de la presente revisión de literatura es conocer la respuesta en producción y composición de leche ante la suplementación de diferentes formas de metionina sintética en ganado lechero.

\section{METIONINA SINTÉTICA EN LA ALIMENTACIÓN DEL GANADO LECHERO}

\section{Aminoácidos como requerimiento nutricional del ganado lechero}

En la nutrición animal existe un patrón ideal de aminoácidos absorbidos para cada una de las funciones fisiológicas (NRC 2001). Los animales superiores requieren un núcleo de nueve aminoácidos para mantenimiento y propósitos productivos, a saber: lisina, histidina, leucina, isoleucina, valina, metionina, treonina, triptofano y fenilalanina (D' Mello 2003). La necesidad de estos aminoácidos surge de la inhabilidad de todos los animales para sintetizar el correspondiente esqueleto de carbono. Los animales monogástricos reciben estos aminoácidos vía dieta, y los rumiantes pueden, además de esa misma vía, adquirir cantidades substanciales de estos $A A$ a través de la digestión de microorganismos sintetizados en el rumen (NRC 2001).

La absorción de AA, provista por la proteína de sobrepaso, el nitrógeno endógeno y la síntesis de proteína microbial, es esencial para la construcción de tejido y proteína láctea, así como en menor medida, también para la síntesis de otros metabolitos del 
cuerpo (NRC 2001). Así por ejemplo, la metionina es importante para producir formilmetionina (fMet) cuya función es iniciar la síntesis de proteína. La L-Met es un aminoácido nutricionalmente indispensable que puede ser usado para la síntesis de proteína e incorporado al ciclo de Met (Martin et al. 2013). También produce Sadenosilmetionina, donador de grupos metilo que participan en reacciones para la biosíntesis de lípidos y otros compuestos que están envueltos en el transporte de lípidos hacia el torrente sanguíneo (Patterson et al. 1988, D' Mello 2003).

De acuerdo con el NRC (2001), los requerimientos de aminoácidos para ganado lechero no son conocidos con mucha certeza. Desde el año 1981, se han realizado aproximaciones de los requerimientos utilizando un modelo factorial, a partir de los requerimientos netos de proteína para mantenimiento, crecimiento, preñez y lactancia. De esta forma, para obtener un máximo contenido y rendimiento de la proteína en la leche, se ha establecido que la Lis debe contribuir alrededor de un 7,0 \% del total de proteína metabolizable (PM), mientras que la Met un 2,5\%. De ahí la importancia de identificar la composición de aminoácidos en los alimentos utilizados en ganado lechero y suplir las deficiencias haciendo uso de AA sintéticos.

\section{Producción de aminoácidos sintéticos}

Debido a la necesidad de evitar la degradación de los AA a nivel ruminal de manera que impacten a nivel intestinal, se han desarrollado diversas tecnologías que protejan los aminoácidos, como recubrimientos con polímeros. Sin embargo, esto ha enfrentado algunas dificultades ya que hay una falta de resistencia de los productos encapsulados y la reactividad de algunos aminoácidos con el recubrimiento de polímero es inestable. Esto provoca la ruptura de las partículas en el rumen después de la masticación, sumado a las inconsistencias de degradación post ruminal del polímero (Robinson 2010).

La forma más antigua de proteger proteínas y aminoácidos es el tratamiento por calor. Este proceso consiste en aplicar un moderado nivel de calor promoviendo la reacción de Maillard, donde los azúcares presentes crean un enlace que los microorganismos del rumen no pueden romper (Chalupa 1975). Actualmente existen otras alternativas de protección, como la protección con taninos, polímeros, la quelación, o encapsulación con grasas, así como el uso de aminoácidos análogos (Metcalf 2001).

La protección con polímeros es pH-sensitiva y es diseñada para mantener su integridad estructural en el $\mathrm{pH}$ normal encontrado en el rumen. El bajo $\mathrm{pH}$ del 
abomaso dispara el desdoblamiento de la protección, permitiendo la liberación del aminoácido para la absorción.

Todos los aminoácidos utilizados en la síntesis de proteínas pueden estar en la configuración L-, sin embargo los animales son capaces de utilizar algunos enantiómeros D-. En efecto, la generalizada comercialización de suplementos para dietas con metionina se ha logrado con la mezcla racémica (D' Mello 2003), como por ejemplo la forma química del DL isómero ácido 2-hidrox 1-4-metiltio butanóico (HMTBA) o el éster de isopropil (HMBi) (Figura 1).

DL-Metionina<smiles>CSCCCC(N)C(=O)O</smiles>

Hidroxianálogo de Metionina<smiles>CSc1ccc(CCC(=O)O)cc1</smiles>

Figura 1. Estructura de DL-metionina e hidroxianálogo de metionina (Patterson y Kung 1988).

El HMTBA es producido por un proceso simple, ya que no es necesario insertar un grupo amino en la molécula. Los aminoácidos análogos o derivados han sido evaluados por su habilidad de resistir la degradación microbial (Papas et al. 1984).

\section{Degradación, absorción y metabolismo de aminoácidos sintéticos}

La acción de adicionar aminoácidos sintéticos a la dieta del rumiante no garantiza su total absorción a nivel intestinal, ya que una porción de éstos serán degradados a nivel ruminal. En el caso de los análogos de la Met, una vez adicionados e ingeridos, el $60 \%$ será utilizado en el rumen donde estimulará la síntesis de proteína microbial, el 40\% remanente abandona el rumen con la fase líquida de la ingesta y será absorbido a lo largo del tracto digestivo (Klangnok et al. 2011). Por lo que la fuente degradable de Met puede tener diferentes mecanismos de acción. 
El $60 \%$ que se utiliza a nivel ruminal estimulará el crecimiento de microorganismos que pueden incrementar la digestión de los alimentos (Noftsger et al. 2005). Sin embargo, el impacto de HMBTA o $\mathrm{HMBi}$ sobre la digestión de los alimentos a nivel ruminal parece ser variable y poco clara (Martin et al. 2013). Diversas investigaciones in vitro e in vivo han mostrado tanto efectos positivos (Noftsger et al. 2005) como negativos en la fermentación y digestibilidad de la fibra a nivel ruminal (Noftsger y St-Pierre 2003).

Patton et al. (1970) sugiere que la suplementación con ésteres de isopropil (HMBi) causa un incremento en la biomasa de protozoarios. La Met funge como donador de metilos para la producción de fosfatidilcolina, por lo que se considera que estos microorganismos son el primer suplidor de fosfatidilcolina. Esta es una importante molécula utilizada en el empaque de ácidos grasos dentro de lipoproteínas de baja densidad y quilomicrones. Algunos ácidos grasos de la leche provienen de los quilomicrones y lipoproteína de baja densidad (Yao y Vance 1988).

En cuanto a los aminoácidos protegidos, se considera que poseen una mayor biodisponibilidad intestinal que las fuentes análogas. Dichos resultados se han encontrado evaluando concentraciones plasmáticas de Met cerca de la ubre y relacionándolas con concentraciones de proteína verdadera en leche (Rulquin et al. 2006).

Posteriormente, la cantidad de aminoácido que sobrepasa el rumen llega al intestino y es absorbida hacia la vena porta. El hígado será el primer órgano en reconocer el aminoácido, ya que éste es el sitio catabólico de algunos AA esenciales como metionina, histidina, fenilalanina y tirosina (Berthiaume et al. 2006).

Después de la absorción, la utilización biológica de HMTBA se basa en su conversión a L-Met. La HMTBA será convertida a 2-oxo, 4-metiltiobutanoato (también producido por desaminación de D-Met) seguida por una aminación a L-Met (Martin et al. 2013). La forma oxidada de L-HMTBA existe predominantemente en los peroxisomas del hígado y riñón mientras que la deshidrogenasa de D-HMTBA es una enzima mitocondrial que se encuentra en la mayoría de los tejidos (McCollum et al. 2000).

De acuerdo con Lapierre et al. (2011), el hígado se encarga de extraer una porción considerable de la HMTBA absorbida (34\%); sin embargo, no existen reportes sobre el destino metabólico de HMTBA en vacas lecheras, donde la demanda de Met es exacerbada para una producción de leche sustentable. Por otra parte Berthiaume et al. (2006), reportan que en la lactancia temprana de vacas lecheras la remoción hepática de Met varía entre 30 y $40 \%$ de la absorción portal neta. 
Por lo que para lograr un mejor entendimiento de la relación entre suplir AA y su salida en proteína láctea, se necesita más información en metabolismo post absorbible de aminoácidos y su impacto en la eficiencia de convertir AA absorbidos en proteína láctea (Berthiaume et al. 2006).

Una vez absorbidos los aminoácidos a través del intestino, estarán disponibles para todas las células del cuerpo para la síntesis de proteínas. Los aminoácidos en el torrente sanguíneo son los precursores directos para la síntesis de la caseína, principal proteína láctea, en las células secretoras de la glándula mamaria (Frandson et al. 2003).

El adicionar Met en una dieta deficiente permite mejorar el perfil de AA absorbidos resultando en un incremento en la síntesis de proteína, debido a que cuando un $A A$ esencial es absorbido en el perfil de AA como requerimiento del animal, el requerimiento por el total de $A A$ es reducido y la eficiencia de $A A$ para la síntesis de proteína es maximizado, mecanismo que permite disminuir la excreción de nitrógeno al ambiente (NRC 2001).

\section{Respuesta ante el uso de metionina y lisina sintética}

Desde el año 1984 se registran investigaciones en donde se mide la respuesta en producción y composición láctea ante el suministro de aminoácidos de sobrepaso (lisina o metionina inertes al rumen, LPR y MPR, respectivamente), hidroxianálogos de Metionina (HMBTA o $\mathrm{HMBi}$ ), o aminoácidos simples, ya sea adicionados a la ración o ajustando la relación Lis:Met en la dieta. El resumen de algunas investigaciones y los resultados más relevantes se presentan en el Cuadro 1.

Del total de artículos analizados, $67 \%$ de aquellos que buscaban el propósito de incrementar la producción o composición láctea tuvieron éxito ante el suministro aminoácidos sintéticos, ya fuera Met o Met+Lis. Del total de artículos en donde se buscaba disminuir la cantidad de PC en la ración total y mejorar la eficiencia de $\mathrm{N}$ adicionando aminoácidos, el 50\% tuvo éxito ante el suministro de este aditivo.

En general, los resultados que se muestran en el Cuadro 1, no han sido constantes en las diversas variables de respuesta evaluadas. Zanton et al. (2014) realizaron un metaanálisis con diversos trabajos que estudiaron la variable de respuesta al suplementar con fuentes de Met sintética o con infusión postruminal de Met y concluyeron que no existen respuestas significativas entre el rendimiento lácteo y la suplementación de 
Met. Sin embargo, existe una tendencia de incremento en la producción láctea cuando las vacas fueron suplementadas con HMTBA.

En el mismo meta-análisis se concluye que el rendimiento de grasa láctea fue significativamente afectado por la suplementación de HMBTA en comparación con las fuentes de metionina protegida (MPR). En tanto, la suplementación de HMBTA o HMBi no ha sido constante en la concentración y rendimiento de proteína láctea en comparación con MPR (Zanton et al. 2014).

Diversos autores han concluido que las inconsistencias en respuestas a la utilización de aminoácidos sintéticos se deben al estado de la lactancia (Schwab et al. 1992), suministro de metionina de la dieta basal (Rulquin et al. 1993), adecuación de Lis en la dieta (NRC 2001) y potencial genético de los animales (Klangnok et al. 2011).

Noftsger et al. (2005) menciona que la falta en respuestas en producción de proteína láctea y rendimiento en la producción de leche en la mayoría de los trabajos pueden indicar que HMTBA no está escapando del catabolismo microbial en significantes concentraciones para ser usado a nivel postruminal.

En otro aspecto, Klangnok et al. (2011) no encontraron respuestas positivas al adicionar HMTBA debido a un bajo potencial genético de las vacas utilizadas, por lo que el incremento en el suministro de metionina al duodeno no afectó el rendimiento lácteo ni composición láctea.

Otro factor influyente en los resultados de desempeño del AA es la duración del período de evaluación del efecto. Por ejemplo, Phipps et al. (2008) concordaron que el empleo de un cuadrado latino de 4 semanas como diseño experimental puede afectar la respuesta. En experimentos de larga duración, St-Pierre y Sylvester (2005) encontraron un incremento en la producción de leche atribuible a una respuesta progresiva, la cual alcanzó significancia a la semana 11 de 17 experimentales. 
Cuadro 1. Resumen de revisión de literatura sobre respuestas ante el uso de AA sintéticos en vacas lecheras.

\begin{tabular}{|c|c|c|c|}
\hline Fuente de $A A$ & Metodología & Respuesta & Referencia \\
\hline $\begin{array}{l}\text { Met inerte al } \\
\text { rumen (MPR) }\end{array}$ & $0,157,315,472,639 \mathrm{mg} / \mathrm{kg} \mathrm{MS}$ & $\begin{array}{l}\text { Concentración de Met en plasma sanguíneo siguió } \\
\text { tendencia lineal. Suplementación incrementó } \\
\text { consumos y ganancias diarias de peso en vacas } \\
\text { lecheras. }\end{array}$ & $\begin{array}{l}\text { Papas } \\
\text { et al. } 1984\end{array}$ \\
\hline DL-Met simple & Niveles crecientes de 0,15 y 30 g/día & $\begin{array}{l}\text { Alimentación con } 30 \mathrm{~g} / \mathrm{día} \text { incrementó la } \\
\text { producción láctea en } 2,5 \mathrm{~kg} \text {, no detectaron } \\
\text { diferencia en leche residual ni producción de } \\
\text { grasa. El contenido graso en leche residual tuvo } \\
\text { efectos significativos al utilizar } 15 \mathrm{~g} / \text { día. }\end{array}$ & $\begin{array}{l}\text { Dormond } \\
\text { et al. } 1990\end{array}$ \\
\hline $\begin{array}{l}\text { Hidroxianálogo de } \\
\text { Met (HMTBa) }\end{array}$ & $0,10,20$ y 30 g/día & $\begin{array}{l}\text { No se encontraron diferencias significativas en } \\
\text { producción y composición láctea, la tendencia fue } \\
\text { negativa al incrementar el nivel del aditivo en las } \\
\text { variables estudiadas. }\end{array}$ & Arroyo 1992 \\
\hline $\begin{array}{l}\text { DL-Met y L-Lis } \\
\text { simples }\end{array}$ & $\begin{array}{l}\text { Dietas a base de maíz suplementadas } \\
\text { con harina de soya, semilla de } \\
\text { algodón o harina de gluten de maíz, } \\
\text { ante la respuesta de Lis y Met en } \\
\text { cantidades de } 5,6 \mathrm{~g} \text { de DL-Met y } 16,6 \\
\text { g de L-Lis. }\end{array}$ & $\begin{array}{l}\text { En lactancia temprana y tardía la adición de AA } \\
\text { elevó la concentración proteica } 1,0 \mathrm{~g} / \mathrm{kg} \text { de leche. }\end{array}$ & $\begin{array}{l}\text { Armentano } \\
\text { et al. } 1993\end{array}$ \\
\hline
\end{tabular}


Cont. Cuadro 1. Resumen de revisión de literatura sobre respuestas ante el uso de AA sintéticos en vacas lecheras.

\begin{tabular}{|c|c|c|c|}
\hline Fuente de $A A$ & Metodología & Respuesta & Referencia \\
\hline $\begin{array}{l}\text { Met y Lis } \\
\text { protegidos al } \\
\text { rumen (AAPR) }\end{array}$ & $\begin{array}{l}\text { Los AA fueron adicionados a nivel bajo de PC } \\
(14 \%) \text { para proveer el } 0 \%, 50 \% \text {, } 100 \% \text { y } 150 \% \\
\text { de la eficiencia predicha de Lis y Met, control } \\
18 \% \text { PC sin suplementación AA. }\end{array}$ & $\begin{array}{l}\text { Mayor rendimiento en producción y } \\
\text { composición láctea para el tratamiento } \\
\text { con alta PC. Al suplir AA a bajo nivel de } \\
\text { PC no se afectó consumo de MS, } \\
\text { rendimientos lácteos ni composición, a } \\
\text { excepción del rendimiento en grasa } \\
\text { láctea que tuvo un efecto mayor con } 0 \text { y } \\
150 \% \text { de la eficiencia de Met y Lis. El } \\
\text { porcentaje de proteína y caseína } \\
\text { incrementaron linealmente cuando las } \\
\text { vacas fueron alimentadas con niveles } \\
\text { crecientes de AA protegidos. }\end{array}$ & $\begin{array}{l}\text { Piepenbrink } \\
\text { et al. } 1996\end{array}$ \\
\hline
\end{tabular}

Met $y$ Lis protegidos al rumen (AAPR)

Adición de $12 \mathrm{~g}$ de mezcla que incluía $50 \%$ Lis $+15 \%$ de Met, ante el suministro de somatotropina bovina (bST) en dietas con $17,6 \%$ PC.
Adición de AA incrementó porcentaje de grasa, proteína y sólidos totales en leche. Sin embargo el rendimiento en $\mathrm{Kg}$ de estos componentes no se vio afectado.
Bremmer

et al. 1997
Tratamientos: 1) 86 y $90 \%$ de los requerimientos de Lis y Met, 2) 112 y $103 \%$ de los requerimientos de Lis y Met, 3 ) Tratamiento 1 + Met y Lis protegida al rumen ( $27 \mathrm{~g} /$ día de Lis y $8 \mathrm{~g} /$ día de Met como AA disponibles) y 4) Tratamiento 1 + Met y Lis protegida al rumen a tratamiento 1 (40 g/día de Lis y $13 \mathrm{~g} / \mathrm{día}$ de Met como AA disponibles).
Rendimiento lácteo y porcentaje de proteína láctea fue mayor en tratamiento 4 durante las primeras 8 semanas de lactancia.
$\mathrm{Xu}$

et al. 1998 protegidos al rumen (AAPR) 
Cont. Cuadro 1. Resumen de revisión de literatura sobre respuestas ante el uso de AA sintéticos en vacas lecheras.

\begin{tabular}{ll}
\hline Fuente de $A A$ & Metodología Respuesta Refencia \\
\hline
\end{tabular}

\begin{tabular}{|c|c|}
\hline $\begin{array}{l}\text { Met inerte al } \\
\text { umen (MPR) }\end{array}$ & $\begin{array}{l}\text { Dos niveles de PC }(16,1 \text { y } 18,8 \%) \text { y } \\
\text { suplementación con Met }(0,07 \mathrm{~g} / 100 \mathrm{~g} \text { de } \mathrm{MS}) \\
\text { alcanzando niveles de Lis y Met en PM de } 6,5- \\
6,3 \% \text { y } 1,8-1,7 \% \text { para bajo y alto nivel de PC. }\end{array}$ \\
\hline
\end{tabular}

Incrementó la concentración de proteína con la adición de Met, y disminución conforme incrementaba el contenido de PC. No se encontraron diferencias significativas en producción láctea ni concentración de grasa con el aumento de la proteína. No se encontraron diferencias significativas en sólidos no grasos, lactosa, o caseína.

Leonardi

et al. 2003

El aumento de proteína cruda incrementa nitrógeno ureico en leche por $3,9 \mathrm{mg} / \mathrm{dl}$, sin incrementar los rendimientos lácteos. La suplementación con Met no tuvo un efecto en excreción de $\mathrm{N}$ en orina ni heces.
Met inerte al

rumen (MPR) e

Hidroxianálogos

de Met HMBi y

Utilizando dosis que aportaran $10 \mathrm{~g}$ de Met absorbible comparadas con un control negativo.
Las concentraciones de Met en plasma sanguíneo fueron mayores con el uso de $\mathrm{HMBi}, \mathrm{HMTBA}$ y MPR respecto al control, así como mayores rendimientos en proteína láctea con HMBi y MPR. No se mejoró la disponibilidad de Met para la síntesis de proteína láctea con HMTBA.
Rulquin

et al. 2006 
Cuadro 1. Resumen de revisión de literatura sobre respuestas ante el uso de AA sintéticos en vacas lecheras.

\begin{tabular}{|c|c|c|c|}
\hline Fuente de $A A$ & Metodología & Respuesta & Referencia \\
\hline $\begin{array}{l}\text { Met inerte al } \\
\text { rumen (MPR) }\end{array}$ & 0, 36 y 72 g/día & $\begin{array}{l}\text { No encontraron efectos en rendimiento } \\
\text { lácteo ante la dosis. Encontraron } \\
\text { incremento lineal en el contenido de } \\
\text { proteína verdadera en leche debido a un } \\
\text { aumento en la concentración arterial de } \\
\text { Met. }\end{array}$ & $\begin{array}{l}\text { Berthiaume } \\
\text { et al. } 2006\end{array}$ \\
\hline $\begin{array}{l}\text { Met inerte al } \\
\text { rumen (MPR) e } \\
\text { Hidroxianálogo de } \\
\text { Met (HMBi) }\end{array}$ & $\begin{array}{l}\text { Ajuste de Lis y Met de PM en } 3,0: 1(0,06 \% \text { de } \\
\text { MPR en dieta } 15,5 \% \text { PC), control de } 16,8 \% \text { sin } \\
\text { suplemento de AA. }\end{array}$ & $\begin{array}{l}\text { HMBi incrementó el rendimiento lácteo, } \\
\text { contenido proteico y sólidos no grasos en } \\
\text { todas las dietas con Met suplementada. } \\
\text { Dietas con } 16,8 \% \text { PC sin fuentes de Met } \\
\text { eleva el NUL, excreción urinaria de } \mathrm{N} \text { y } \\
\text { reduce la eficiencia aparente de } \mathrm{N} \text { de } \\
34,5 \% \text { a } 30,2 \% \text {, en comparación con las } \\
\text { dietas que contenían } 15,5 \% \text { PC más AA } \\
\text { de sobrepaso. }\end{array}$ & $\begin{array}{l}\text { Chen } \\
\text { et al. } 2011\end{array}$ \\
\hline $\begin{array}{l}\text { Hidroxianálogo de } \\
\text { Met (HMTBa) }\end{array}$ & 11 g/d y 22 g/día & $\begin{array}{l}\text { No hubo cambios en composición láctea } \\
\text { ni rendimientos. Se incrementaron ácidos } \\
\text { grasos insaturados y disminuyeron } \\
\text { ácidos grasos saturados ante la } \\
\text { suplementación en la composición de } \\
\text { grasa láctea. }\end{array}$ & $\begin{array}{l}\text { Klangnok } \\
\text { et al. } 2011\end{array}$ \\
\hline $\begin{array}{l}\text { Hidroxianálogo de } \\
\text { Met (HMBi) }\end{array}$ & $\begin{array}{l}\text { HMBi }(0,2 \% \text { MS) en vacas lactantes, dieta baja } \\
\text { en PC (13,3\%) en dos dietas basadas en } \\
\text { ensilaje de maíz o ensilaje de otro forraje. }\end{array}$ & $\begin{array}{l}\text { Incrementaron los rendimientos en } \\
\text { sólidos lácteos, caseína y PC. La fuente } \\
\text { de forraje+ HMBi no tuvo efecto sobre la } \\
\text { excreción de } \mathrm{N} \text { en heces, orina o leche. }\end{array}$ & $\begin{array}{l}\text { Whelan } \\
\text { et al. } 2011\end{array}$ \\
\hline
\end{tabular}




\section{CONSIDERACIONES FINALES}

En el mercado de aditivos nutricionales existen diferentes aminoácidos sintéticos que aparentan ser una opción viable para incrementar las concentraciones de grasa y proteína láctea en vacas altas productoras en la lactancia temprana. Sin embargo, se debe tener claro que los resultados de diferentes investigadores han sido inconsistentes en múltiples investigaciones.

Se puede esperar que en el futuro, las nuevas investigaciones y resultados más precisos en los requerimientos de aminoácidos para rumiantes, los mecanismos fisiológicos post absorbibles y la composición de aminoácidos en forrajes, abran una ventana de conocimientos en la que se pueda implementar esta tecnología de una forma más precisa y eficiente.

\section{LITERATURA CITADA}

Armentano, L.E., Swain, S. M., Ducharme, G. A. 1993. Lactation response to ruminally protected methionine and Lisine at two amounts of ruminally available nitrogen. $\mathrm{J}$. Dairy Sci. 76(10): 2963-2969.

Arroyo, J.G. 1992. Utilización de diferentes niveles de MHA (Hidroxianálogo de Metionina) sobre producción y composición de la leche en vacas en pastoreo durante el verano. Tesis presentada para optar por el título de licenciatura en ingeniería agronómica con énfasis en zootecnia. UCR. San Pedro de Montes de Oca, Costa Rica.

Berthiaume, R., Thivierge, M.C., Patton, R.A., Dubreuil, P., Stevenson, M., Mcbride, B.W., Lapierre, H. 2006. Effect of ruminally protected methionine on splanchnic metabolism of amino acids in lactating dairy cows. J. Dairy Sci. 89(5): 1621-1634.

Bremmer, D.R., Overton, T.R., Clark, J.H. 1997. Production and composition of milk from Jersey cows administered bovine somatotropin and fed ruminally protected amino acids. J. Dairy Sci. 80(7): 1374-1380.

Chalupa, W. 1975. Rumen bypass and protection of proteins and amino acids. J. Dairy Sci. 58(8): 1198-1218 
Chen, Z.H., Broderick, G.A., Luchini, N.D., Sloan, B.K., Devillard, E. 2011. Effect of feeding different sources of rumen-protected methionine on milk production and N-utilization in lactating dairy cows. J. Dairy Sci. 94(4): 1978-1988.

D' Mello, J.P. 2003. Amino acids in Animal Nutrition. CABI. Segunda Edición. Oxon United Kindom. 495 pp.

Dormond, H., Rojas, A., Boschini, C. 1990. Efecto de la DL-Metionina sobre parámetros productivos en vacas de doble propósito. Agronomía Costarricense. 14(1): 31-36.

Frandson, R.D., Wilke, W. Lee., Deefails, A. 2003. Anatomy and physiology of farm animals. $6^{\text {th }}$ edition. Blackwell Publishing.

Klangnok, P., Lounglawan, P., Suksombat, W. 2011. Effects of met hydroxy analog (MHA $®)$ supplementation of dairy cow' $s$ diets on milk yield and milk composition. Suranaree J. Sci. Technol. 18(2):99-108.

Lapierre, H.M., Vázquez-Añón, D.P., Dubreuil, G.H., Lobley, G.E. 2011. Metabolism of 2-Hydroxy-4-(methylthio)butanoate (HMTBA) in lactating dairy cows. J. Dairy Sci. $94(3): 1526-1535$.

Leonardi, C., Stevenson, M., Armentano, L. E. 2003. Effect of two levels of crude protein and methionine supplementation on performance of dairy cows. J. Dairy Sci. 86(12): 4033-4042.

Martin, C., Mirande, C., Morgavi, D.P., Forano, E., Devillard, E., Mosoni, P. 2013. Methionine analogues $\mathrm{HMB}$ and $\mathrm{HMBi}$ increase the abundance of cellulolytic bacterial representatives in the rumen of cattle with no direct effects on fibre degradation. Animal Feed Science and Technology. 182(1-4): 16-24.

McCollum, M., Vazquez-Anon, Q.M., Dibner, J.J., Webb, K.E. 2000. Absorption of 2hydroxy-4 (methylthio) butanoic acid by isolated sheep ruminal and omasal epithelia. J. Anim. Sci. 78:1078-1083.

Metcalf, J. 2001. Understanding bypass vegetable proteins. Feed Mix Magazine. 9(4):15-16.

Noftsger, S.M., St-Pierre, N.R., 2003. Supplementation of methionine and selection of highly digestible rumen undegradable protein to improve nitrogen efficiency for milk production. J. Dairy Sci. 86:958-969.

Noftsger, S.M., St-Pierre, N.R., Sylvester, J.T. 2005. Determination of rumen degradability and ruminal effects of three sources of methionine in lactating cows. J. Dairy Sci. 88:223-237.

NRC (National Research Council). 2001. Nutrients Requeriments of Dairy Cattle. 7th ed. Natinal Academy Press. Washington D.C.

Papas, A.M., Sniffen, C.J., Muscato, T.V. 1984. Effectiveness of Rumen-Protected Methionine for Delivering Methionine Postruminally in Dairy Cows. J. Dairy Sci. 67(3): 545-552. 
Patterson, J., L Kung. 1988. Metabolism of DL-methionine and methionine analogs by rumen microorganisms. J Dairy Sci. 71(12): 3292-3301.

Patton, R.A., McCarthy, R.D., Griel, L.C. 1970. Lipid synthesis by rumen microorganisms. II. Further characterization of the effects of methionine. J. Dairy Sci. 53:460-465

Phipps, R.H., Reynolds, C.K., Givens, D.I., Jones, A.K., Geraert, P., Devillard E., Bennett, R. 2008. Short communication: effects of 2-hydroxy-4-(methylthio) butanoic acid isopropyl ester on milk production and composition of lactating Holstein dairy cows. J. Dairy Sci. 91(10):4002-4005.

Piepenbrink, M. S., Overton, T. R., Clark, J. H. 1996. Response of cows fed a low crude protein diet to ruminally protected methionine and Lisine. J. Dairy Sci. 79(9): 1638-46. Oxford. UK. 481p.

Robinson, P.H. 2010. Impacts of manipulating ration metabolizable Lisine and methionine levels on the performance of lactating dairy cows: A systematic review of the literature. Livestock Science. 127(2-3): 115-126.

Rulquin, H., Graulet, B., Delaby, L., Robert, J.C. 2006. Effect of different forms of methionine on lactational performance of dairy cows. J. Dairy Sci. 89(11): 43874394.

Rulquin, H., Pisulewski, P.M., Ve'rite', R., Guinard, J. 1993. Milk production and composition as a function of postruminal lysine and methionine supply: a nutrientresponse approach. Livest. Prod. Sci. 37:69-90.

Schwab, C.G., Bozak, C.K., Whitehouse, N.L., Mesbah, M.M.A. 1992. Amino acid limitation and flow to duodenum at four stages of lactation. 1. Sequence of lysine and methionine limitation. J. Dairy Sci. 75:3486-3502.

ST-Pierre, N.R., Sylvester, J.T. 2005. Effects of 2-hydroxy-4-(methylthio) butanoic acid (HMB) and its isopropyl ester on milk production and composition by Holstein cows. J. Dairy Sci. 88:2487-2497.

Whelan, S.J., Mulligan, F.J., Flynn, B., Mccarney, C., Pierce, K.M. 2011. Effect of forage source and a supplementary methionine hydroxy analog on nitrogen balance in lactating dairy cows offered a low crude protein diet. J. Dairy Sci. 94(10): 5080-5089.

Xu, S., Harrison, J.H., Chalupa, W., Sniffen, C., Julien, W., Sato, H., Fujieda, T. 1998. The effect of ruminal bypass Lisine and methionine on milk yield and composition of lactating cows. J. Dairy Sci. 81(4): 1062-1077.

Yao, Z., Vance, D.E. 1988. The active synthesis of phosphatidyl- choline is required for very low density lipoprotein secretion from rat hepatocytes. J. Biol. Chem. 263:2998-3004.

Zanton, G.I., Bowman, G.R., Vázquez-Añón, M., Rode, L.M. 2014. Meta-analysis of the lactation performance in dairy cows receiving supplemental dietary methionine sources or postruminal infusion of methionine. J. Dairy Sci. 97:1-17. 